\title{
Orang-utans rely on orientation cues and egocentric rules when judging others' perspectives in a competitive food task
}

\author{
Heinz Gretscher ${ }^{\mathrm{a}, *}$, Daniel B. M. Haun ${ }^{\mathrm{a}, \mathrm{b}}$, Katja Liebal ${ }^{\mathrm{c}}$, Juliane Kaminski ${ }^{\mathrm{a}}$ \\ ${ }^{a}$ Max Planck Institute for Evolutionary Anthropology, Leipzig, Germany \\ ${ }^{\mathrm{b}}$ Max Planck Institute for Psycholinguistics, Nijmegen, The Netherlands \\ ${ }^{c}$ Cluster Languages of Emotion, Freie Universität Berlin, Berlin, Germany
}

\section{A R T I C L E I N F O}

Article history:

Received 18 September 2011

Initial acceptance 13 February 2012

Final acceptance 20 March 2012

Available online 12 June 2012

number: 11-00746

\section{Keywords:}

competition

deception

orang-utan

Pongo pygmaeus

social cognition

theory of mind

visual perspective taking
Adopting the paradigm of a study conducted with chimpanzees, Pan troglodytes (Melis et al. 2006, Journal of Comparative Psychology, 120,154-162), we investigated orang-utans', Pongo pygmaeus, understanding of others' visual perspectives. More specifically, we examined whether orang-utans would adjust their behaviour in a way that prevents a human competitor from seeing them steal a piece of food. In the task, subjects had to reach through one of two opposing Plexiglas tunnels in order to retrieve a food reward. Both rewards were also physically accessible to a human competitor sitting opposite the subject. Subjects always had the possibility of reaching one piece of food that was outside the human's line of sight. This was because either the human was oriented to one, but not the other, reward or because one tunnel was covered by an opaque barrier and the other remained transparent. In the situation in which the human was oriented towards one reward, the orang-utans successfully avoided the tunnel that the competitor was facing. If one tunnel was covered, they marginally preferred to reach through the opaque versus the transparent tunnel. However, they did so frequently after initially inspecting the transparent tunnel (then switching to the opaque one). Considering only the subjects' initial inspections, they chose randomly between the opaque and transparent tunnel, indicating that their final decision to reach was probably driven by a more egocentric behavioural rule. Overall the results suggest that orang-utans have a limited understanding of others' perspectives, relying mainly on cues from facial and bodily orientation and egocentric rules when making such judgements.

(c) 2012 The Association for the Study of Animal Behaviour. Published by Elsevier Ltd. All rights reserved.
The capacity to take the visual perspective of others is an integral part of human social interactions, especially when it comes to judging whether, and how, others will perceive and react to events in their environment. Whereas human children excel in fully fledged visual perspective taking from an early age (Flavell et al. 1981; Moll \& Meltzoff 2011), as of yet it remains unclear to what extent this trait is uniquely human or shared with other animal species. The ability to follow the gaze of others, which is thought of as a prerequisite for more sophisticated forms of understanding what others 'see', seems to be widespread in the animal kingdom (primates: Call et al. 2001; ungulates: Kaminski et al. 2005; birds: Loretto et al. 2010; reptiles: Wilkinson et al. 2010). However, evidence for higher-level gaze following that is not explicable in terms of a simple co-orientation response (Povinelli \& Eddy 1996) is scarcer. So far only wolves, Canis lupus (Range \& Virányi 2011), ravens, Corvus corax (Bugnyar et al. 2004) and some primates

\footnotetext{
* Correspondence: H. Gretscher, Max Planck Institute for Evolutionary Anthropology, Deutscher Platz 6, D-04103 Leipzig, Germany.

E-mail address: gretsche@eva.mpg.de (H. Gretscher).
}

(Bräuer et al. 2005; Amici et al. 2009) have been found to follow the gaze of others around barriers, while primates alone have been reported to visually check back with the looker if being unable to trace a potential target in their sight (primate gaze following reviewed in Rosati \& Hare 2009).

Furthermore, there are two major lines of evidence indicating that several species are able to track the attentional state of others. One line of support comes from investigations of communicative interactions. For instance, dogs, Canis familiaris (Gácsi et al. 2004), dolphins, Tursiops truncates (Xitco et al. 2004), horses, Equus caballus (Proops \& McComb 2010), multiple primate species (Povinelli \& Eddy 1996; Kaminski et al. 2004; Liebal et al. 2004a; Hattori et al. 2010; Tempelmann et al. 2011), and even birds such as ravens (Pika \& Bugnyar 2011) are known to address their begging behaviour and visual gestures predominantly to attentive recipients. There is also some evidence that chimpanzees, Pan troglodytes, vocalize more frequently when requesting food from an inattentive human, possibly as a strategy to draw the human's attention to themselves (Hostetter et al. 2001, 2007; Leavens et al. 2010).

A second line of evidence stems from investigations of competitive situations. As recently demonstrated, food competition 
paradigms seem to be ecologically more valid to many animal species and thus more likely to elicit complex cognitive abilities (Hare 2001). Various animals tested in such situations take into account the attentional focus of conspecifics and humans, avoiding food that is visible to their competitor (i.e. depending on paradigm, either being more reluctant to retrieve such food or instead preferring alternative food invisible to their competitor), among them species as distantly related as dogs (Call et al. 2003; Bräuer et al. 2004), goats, Capra hircus (Kaminski et al. 2006), jackdaws, Coloeus monedula (von Bayern \& Emery 2009), scrub-jays, Aphelocoma coerulescens (Emery \& Clayton 2001), starlings, Sturnus vulgaris (Carter et al. 2008), and several primates (Flombaum \& Santos 2005; Bräuer et al. 2007; Burkart \& Heschl 2007; Sandel et al. 2011). However, it remains unclear whether the subjects in these tasks really consider what the competitor can 'see'. As the competitor might appear to be absent when individuals move behind a barrier (or overt cues such as visibility of the eyes and eye aversion differed between conditions), the subjects' choice may in fact be egocentrically based. Going somewhat beyond that, chimpanzees and corvids apparently not only understand what another individual can see in the present but also what others have seen in the past (Hare et al. 2001; Bugnyar \& Heinrich 2005; Dally et al. 2006; Kaminski et al. 2008; Bugnyar 2011).

Finally, some animals also actively conceal visual information from competitors (Dally et al. 2004; Hare et al. 2006; Melis et al. 2006). For instance, Dally et al. (2004) found that scrub-jays who were observed by potential pilferers preferred to cache food in a tray that was positioned in the shade over caching in a tray that was well lit (whereas exhibiting no such preference if caching in private). Notably, observers were equally well visible from both trays, ruling out the idea that the scrub-jays were using immediate perceptual cues to solve the task. Similarly Hare et al. (2006) reported chimpanzees to actively conceal the theft of contested food from the view of a human competitor. In the critical experimental conditions of this study, the two food pieces that the chimpanzees could choose from were either positioned such that the human could see both or none. However, one approach route provided cover from the competitor's view whereas the second one did not. In one condition this was because the competitor's face was oriented towards one side but not the other. In a second condition subjects could approach from behind either a transparent barrier or an opaque occluder. In the third condition one side was obstructed by a barrier that fully shielded their approach while the other side was occluded by a (split) barrier that only partially shielded their approach (allowing the human to see the lower body parts of the approaching chimpanzee). In all conditions the chimpanzees consistently chose to approach the contested food via the hidden route (whereas they did not when no competitor was present). Although the chimpanzees could not succeed in the latter two conditions by merely avoiding the route that the competitor was looking at, nevertheless it could be the case that they were simply avoiding the side that allowed them to see the human's face or body parts. To rule this out, Melis et al. (2006) conducted a study in which both pieces of food were visible to the competitor while each of the approaching paths leading to the food was completely hidden from her view. Hence, the competitor was completely hidden from the chimpanzees when they were trying to retrieve the reward. However, the competitor could potentially see their attempt to steal the food, depending on whether they chose to reach through a clear or opaque tunnel. Thus the chimpanzees had to 'imagine' what the competitor could see from her perspective. Similarly, as in the study of Hare et al. (2006), the chimpanzees preferentially chose to reach through the opaque tunnel.

In summary, it seems that although many animals show some basic sensitivity to what others see (either indicated by their ability to follow the gaze of others or taking into account the attentional state of others), as of yet the most conclusive evidence for perspective-taking-like capacities comes from experiments with corvids and great apes. However, this might be the result of a focus of research on relatively few species. This discrepancy precludes us from drawing firm conclusions about the evolutionary trajectory of this skill. For instance, most of the evidence for great apes' perspective taking stems from studies with chimpanzees, whereas much less is known about the other great ape species. As chimpanzees (along with bonobos, Pan paniscus) are the closest relatives to humans, and because they also live in highly complex social groups, their perspective-taking skills might be unique (or at least be most profound) among great apes. However, the trait might be evolutionarily more ancient and therefore also present in the other great ape genera. As orang-utans, Pongo pygmaeus, are phylogenetically the most distant of all great apes from humans as well as the least sociable anthropoid primate species, they represent the ideal test case to resolve this issue.

Orang-utans are the most arboreal great ape species. Their lineage split from that of the other great apes 12-16 million years ago (Locke et al. 2011). Orang-utan life history is characterized by a long life span (35-45 years in the wild, more than 55 years in captivity), slow development and long interbirth intervals (with 8 years the longest among mammals), which might be linked to prolonged learning (Delgado \& van Schaik 2000). Indeed, evidence from the wild indicates that orang-utan behaviour and tool use are to some extent socially transmitted (van Schaik et al. 2003; Krützen et al. 2011). Apart from mothers and their dependent offspring, orang-utans spend most of their time alone. However, during periods of high food abundance they sometimes form small feeding aggregations as well as travel bands (Sugardjito et al. 1987; te Boekhorst et al. 1990). Therefore, orang-utan social structure is thought of as an individual-based fission fusion system (van Schaik 1999).

As yet, evidence for perspective taking in orang-utans is sparse. In following the gaze of others around barriers, orang-utans seem to be less sensitive to the features of objects obstructing the gazer's line of sight than are the African great ape species (Okamoto-Barth et al. 2007). When requesting food from a human experimenter who had the food in front of her but was orientated away from the subject, the orang-utan reliably moved into the experimenter's line of sight (Liebal et al. 2004b). If the location of the food did not correspond with the experimenter's body orientation, orang-utans (unlike chimpanzees and bonobos) occasionally either stayed where they were or manoeuvred behind her and frequently gestured even though the experimenter was unable to see them. By contrast, Shillito et al. (2005) reported that a single female was highly sensitive to the visual states of humans who she directed to desirable objects beyond her reach. Furthermore, orang-utans 'point' more frequently to the location of a hidden tool, if the helper needing the tool to retrieve food for them is ignorant of its whereabouts (Zimmermann et al. 2009). In summary, the existing results are inconsistent, and no study to date has explored orang-utans' understanding of others' visual perspectives in a competitive context.

By adopting the paradigm of Melis et al. (2006) for the present study, our goal was to close this gap, thereby providing more comparative data to trace the evolutionary history of (human) perspective taking. In this task, subjects retrieved one of two pieces of food, both of them positioned in front of two opposing Plexiglas tunnels. Although subjects could approach either reward unseen, only one reward could be reached without the human seeing. In one condition this was because the human was oriented to one but not the other reward. In a second condition the competitor faced both rewards, but one of the two tunnels was covered by an opaque barrier, shielding the orang-utans' grasp from the human's view. 


\section{METHODS}

\section{Subjects}

The subjects were 13 Bornean orang-utans (eight females and five males; see Table 1) housed at the Orangutan Care Centre and Quarantine in Pasir Panjang, Kalimantan Tengah, Indonesia. The orang-utans' ages ranged from 7.5 to 12.0 years (average age $8.9 \pm 1.3$ years). All of them were born in the wild and came to the sanctuary after being confiscated at an early age (range $0.5-4.0$ years; average age $1.6 \pm 1.1$ years) as a result of the logging of rainforest and the trade of orang-utans for pets. Once the apes arrived at the sanctuary, they were raised by human caretakers together with peers until they were old enough to join a social group with mates of their respective sex who were roughly the same age. During the night, the groups were housed in indoor enclosures. During the day, the orang-utans were regularly allowed to forage in a nearby area of tropical forest. The orang-utans were fed various fruits, vegetables and cereals three times a day and were not food deprived for testing at any time. Water was available ad libitum.

\section{Experimental Set-up}

Subjects were tested individually in a testing area consisting of five connected cages (each with a base area of $1.5 \times 1.5 \mathrm{~m}$ ) that were arranged in a U-shaped manner, forming a testing booth (see Fig. 1). Two holes $(10 \times 5 \mathrm{~cm})$ in the centre of the cages at the end of each arm of the $U$ (henceforth 'target cages') led to the inner booth area. Plexiglas tunnels $(25 \times 25 \mathrm{~cm}$ and $21 \mathrm{~cm}$ high $)$ were fixed to the booth side of the cage walls in front of the holes. A Plexiglas flap was fixed inside each tunnel $24 \mathrm{~cm}$ away from the hole. The flaps had to be pulled open to reach a food reward placed on a wooden platform $(25 \times 10 \mathrm{~cm})$ that was attached in front of each of the tunnels. The experimenter (E) sat on a wooden box positioned $1.2 \mathrm{~m}$ away from the middle cage, centred within the booth, between both Plexiglas tunnels. A small plastic bin $(8 \times 5 \mathrm{~cm}$ and $5 \mathrm{~cm}$ high) was fixed to the mesh of the middle cage. Between trials E smeared a food reward (e.g. honey) on the bin to attract subjects to the frontal middle of the central cage (from where they were able to see $\mathrm{E}$ as well as the Plexiglas tunnels and both rewards).

During warm-up trials the wire mesh walls surrounding the tunnels of the target cages were covered with wooden plates, whereas the wire mesh of the central cage remained uncovered. For

Table 1

Sex, age, and warm-up performance of subjects

\begin{tabular}{llcl}
\hline Name & Sex & Approx. age & Warm-up success \\
\hline Ari & Male & 7.5 & Session 2 \\
Cabang & Female & 8.5 & Session 1 \\
Claire & Female & 9.5 & Session 1 \\
Edwin & Male & 8.5 & Session 1 \\
Eric* & Male & 7.5 & - \\
Galih & Male & 7.5 & Session 1 \\
Imas & Female & 7.5 & Session 2 \\
Jill* & Male & 6.0 & - \\
Kraba & Female & 10.0 & Session 1 \\
Lanang & Male & 8.5 & Session 1 \\
Maxene* & Female & 8.5 & - \\
Mercedes & Female & 9.5 & Session 1 \\
Noni & Female & 12.0 & Session 1 \\
Osborne & Female & 9.0 & Session 2 \\
Rowland & Male & 7.5 & Session 1 \\
Sallie & Female & 10.0 & Session 2 \\
Yoris* & Male & 8.5 & - \\
\hline
\end{tabular}

* Did not pass the pretest criterion. the actual experiment additional visual barriers were added to the general set-up. All three cage walls facing the booth were entirely covered with wooden boards, occluding the approaching paths to both target cages. The only openings that remained uncovered were the two holes leading to the interior of the tunnels and a narrow gap in the middle of the central cage (width $17 \mathrm{~cm}$, height $60 \mathrm{~cm}$, starting $25 \mathrm{~cm}$ above the cage floor). The latter allowed the orang-utans to feed from the bait bin and to observe the complete set-up within the booth. To make it more difficult for the orangutans to spot E (or parts of E's body) through the tunnel openings, stripes of opaque adhesive tape (creating a band $10 \mathrm{~cm}$ wide) were stuck to the tunnel walls over the cage holes.

\section{General Procedure}

The general procedure for warm-up and experimental trials was as follows. While subjects were feeding from the bait (e.g. the honey), E placed a reward (e.g. a peanut) in front of each of the tunnels. Then he sat down opposite the subject in a position dictated by the experimental condition (see below). A trial started after subjects had finished eating the bait and left their central (starting) position. To reach the food, subjects had to approach either of the two target cages and pull open the flap door of the respective tunnel. If they opened the flap door in view of $\mathrm{E}, \mathrm{E}$ immediately retracted both food rewards and banged on the opened tunnel with a small wooden stick (to further emphasize the competitive nature of the task). If the subjects approached and pulled open the flap of the tunnel that was out of E's line of sight, they were allowed to retrieve the reward. A trial ended either when the subjects retrieved the food or after $90 \mathrm{~s}$ had passed without the subject opening either of the tunnels.

\section{Warm-up trials}

Before testing, all subjects were familiarized with the general set-up and the competitive nature of the task in a series of warmup trials. In the warm-up trials E turned his head and body towards either of the respective sides, looking at one of the tunnels. If the subjects opened the flap door in front of E, E withdrew both food rewards before the subjects could reach it. If the subjects pulled open the flap of the tunnel at E's back, they were allowed to retrieve the reward. Only subjects who had successfully retrieved four rewards in a row (within a maximum of 36 trials, split into two sessions on two consecutive days) were chosen to participate in the study (see Table 1 ).

\section{Experimental trials}

For the actual experiment additional visual barriers were added, so that the approaching paths to both tunnels were completely occluded (see above). During testing subjects experienced three different conditions in a within-subject design (see Fig. 2):

Body orientation condition (BO). As in the warm-up trials, both tunnels remained transparent and $\mathrm{E}$ was oriented towards one but not the other reward.

Hidden-visible condition (HV). E sat facing the central cage, holding the stick in both hands while looking straight ahead (but never establishing direct eye contact with the subject). One of the tunnels was opaque (covered by a blue plastic sheath) and the other remained transparent.

Nonsocial control (NS). To investigate whether the orang-utans had a general preference for one of the tunnels, we conducted a control condition identical to the hidden-visible condition except that $\mathrm{E}$ left the testing area after placing the food rewards in front of the 


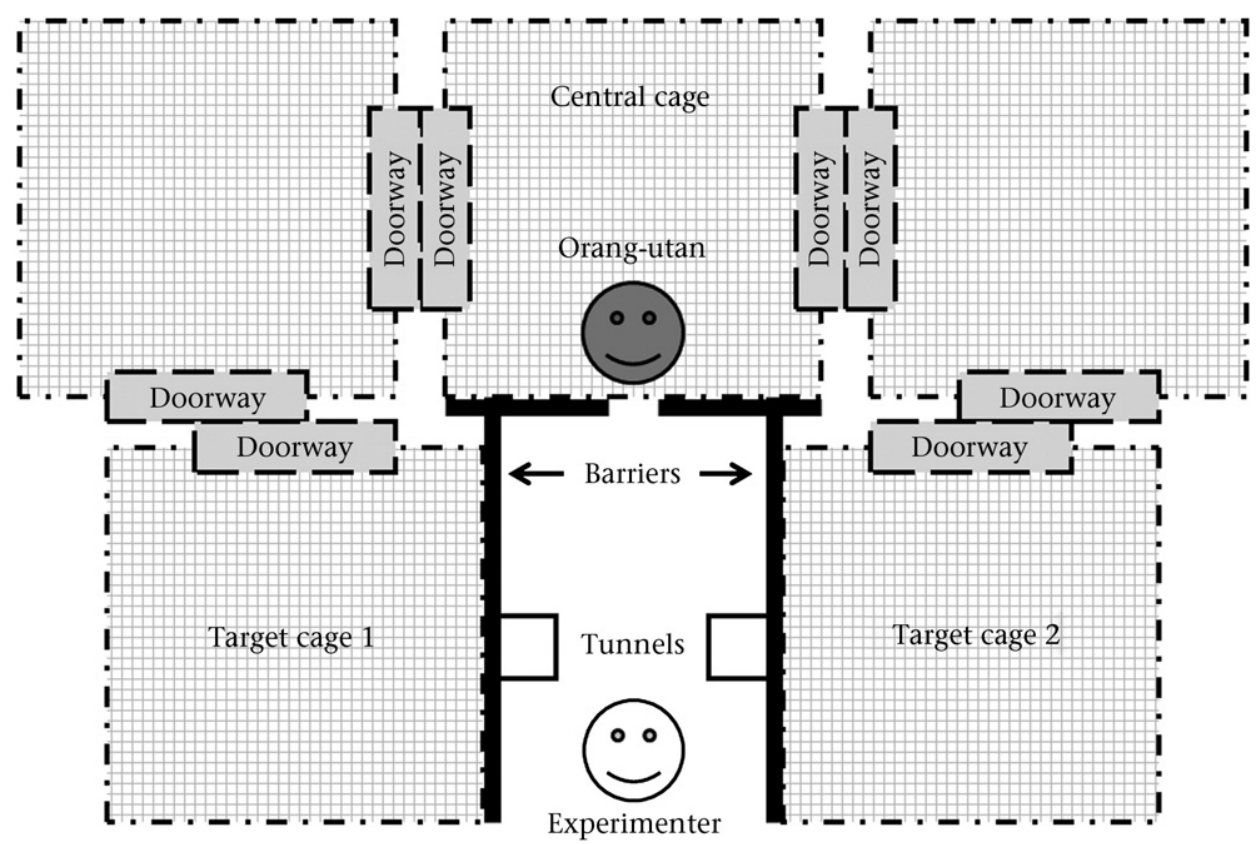

Figure 1. Experimental set-up.

tunnels. Trials ended with E returning after $90 \mathrm{~s}$ or when both rewards had been retrieved.

Subjects received a total of 54 trials ( 18 per condition) split into two daily sessions of nine trial-triplets. Sessions were presented on consecutive days. Each triplet was composed of one trial per condition. Owing to lack of motivation, with one subject (Imas) the second daily session had to be interrupted for a number of hours, and with two others (Kraba, Cabang) the trials had to be split into three daily sessions. The location of the opaque (and the transparent) tunnel was counterbalanced such that in half of the trials the opaque tunnel was on the left whereas in the other half it was on the right. The location of the opaque tunnel was also semirandomized such that the reward could not be retrieved on the same side in more than two consecutive trials. The same was true for the orientation of $\mathrm{E}$ in the body orientation condition.

\section{Coding and Analysis}

All trials were recorded by three digital camcorders from different angles and subsequently scored from the video files. We

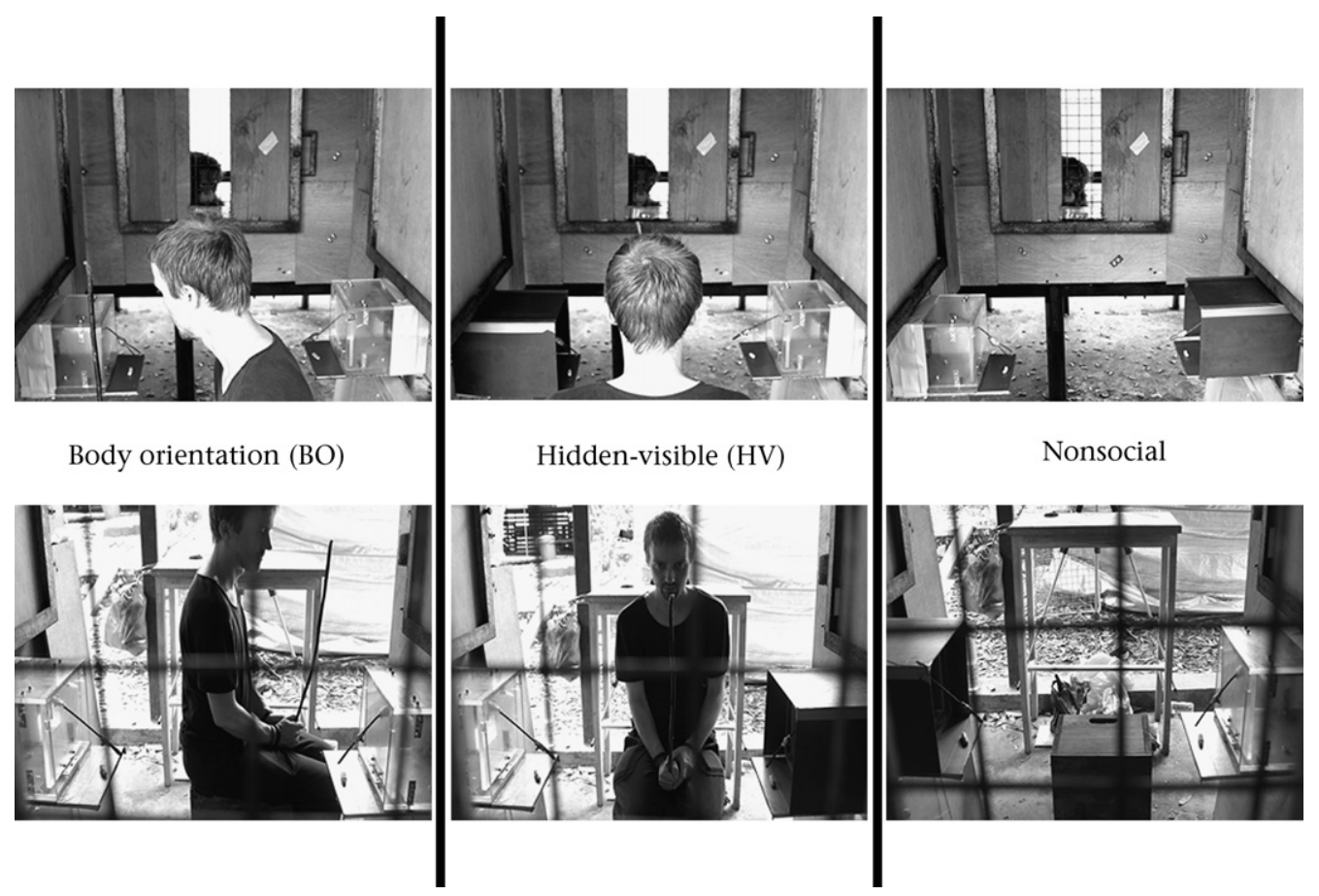

Figure 2. Experimental conditions. Upper row: booth side view; lower row: ape side view. 
coded from which tunnel the subjects chose to retrieve the (first) reward. A 'choice' was defined as pulling open the door flap of the respective tunnel. We also coded which sides the subjects inspected before making their choice (or running out of time). An 'inspection' was scored when subjects either looked through a tunnel opening or reached through the opening, as far as their wrists. We differentiated between two different types of inspections. The first tunnel subjects chose to inspect was coded as 'initial inspection'. If they inspected a tunnel and then approached and inspected the tunnel on the opposite side, the (latter) inspection was coded as a 'side switch'. Finally, we coded how much time subjects spent in total with the 'initial inspection'. The total time was calculated by adding the duration of all single inspection events (i.e. looks and reaches), starting with the first inspection of one tunnel and ending with the pulling open of the tunnel's door flap or switching to the opposite tunnel. A second observer scored $20 \%$ of the trials to assess the interobserver reliability of performance. Interobserver reliability for subject's choice, initial inspections and side switches was excellent (Cohen's $\kappa=0.99$, $\kappa=1.00$ and $\kappa=0.99$, respectively). The Spearman's correlation coefficient for the interobserver reliability of initial inspection times was $\rho=0.64(P<0.001)$. First we compared the total number of trials in which subjects refrained from opening any tunnel across conditions, plus, for each condition separately, whether subjects were more likely to refuse to open the tunnel if (last) inspecting the in-view or out-of-sight tunnel. For subjects' choice and initial inspections, we compared subjects' preference for the out-of-sight tunnel across conditions as well as against chance. As subjects did not make a choice or inspect any tunnel in some trials, the respective results were analysed using proportions. For side switches we compared the total number of trials in which subjects switched sides (at least once) across conditions; plus, for each condition separately, whether subjects were more likely to switch sides depending on the tunnel they had initially inspected. For initial inspection times, we compared the mean times across conditions, as well as within each condition, whether inspection times differed depending on which tunnel the subjects inspected. For all behavioural measurements, we additionally compared subjects' performance in the first nine trials with that in the last nine trials to check for learning effects. Because the data did not satisfy the criteria of normality of distribution and homogeneity of variance (based on Shapiro-Wilk and Levene's test), and as generalized linear mixed models come along with some clear issues regarding the reliability of the $P$ values (Bolker et al. 2009), we chose nonparametric Wilcoxon and Friedman tests as the most simple and reliable. Owing to the small number of subjects, exact probabilities were calculated for all Wilcoxon tests. Unless otherwise stated, all reported $P$ values are two tailed.

\section{RESULTS}

\section{Subjects' Choice}

A first look at the (absolute) number of trials in which subjects refrained from making a choice (see Table 2 ) indicated a difference between conditions (Friedman test: $\chi^{2}=10.889, \quad N=13$, $P=0.004$ ). Post hoc tests revealed that subjects refused to choose most frequently in the HV condition (Wilcoxon signed-ranks test: HV-BO: $T_{+}=33.5, N=8, P=0.039 ; \mathrm{HV}-\mathrm{NS}: T_{+}=28.0, N=7$, $P=0.016)$. The NS and the BO condition did not differ from each other $\left(T_{+}=10.0, N=4, P=0.125\right)$. In the HV condition, subjects were more likely to refuse to choose after (last) inspecting the tunnel in E's sight $\left(T_{+}=26.0, N=7, P=0.047\right)$. In the BO condition, subjects' reluctance to make a choice did not differ depending on whether they (last) inspected the transparent or opaque tunnel
Table 2

Number of trials with side switches

\begin{tabular}{|c|c|c|c|c|c|c|}
\hline \multirow[t]{2}{*}{ Name } & \multicolumn{3}{|c|}{ No choice } & \multicolumn{3}{|c|}{ Side switches } \\
\hline & BO & HV & NS & BO & HV & NS \\
\hline Ari & 0 & 0 & 0 & 0 & 1 & 0 \\
\hline Cabang & 1 & 9 & 0 & 1 & 3 & 0 \\
\hline Claire & 0 & 1 & 0 & 1 & 1 & 0 \\
\hline Edwin & 1 & 0 & 0 & 0 & 2 & 0 \\
\hline Galih & 0 & 0 & 0 & 1 & 2 & 0 \\
\hline Imas & 0 & 3 & 0 & 6 & 8 & 0 \\
\hline Kraba & 2 & 4 & 0 & 1 & 0 & 0 \\
\hline Lanang & 0 & 2 & 0 & 0 & 9 & 0 \\
\hline Mercedes & 2 & 3 & 0 & 1 & 1 & 0 \\
\hline Noni & 0 & 0 & 0 & 0 & 0 & 0 \\
\hline Osborne & 0 & 0 & 0 & 3 & 5 & 0 \\
\hline Rowland & 0 & 1 & 0 & 0 & 4 & 0 \\
\hline Sallie & 0 & 0 & 0 & 0 & 0 & 0 \\
\hline Mean & 0.5 & 0 & 1.8 & 1.2 & 3.0 & 0.0 \\
\hline
\end{tabular}

$\left(T_{+}=1.0, N=1, P=1.000\right)$. The number of trials without any choice did not differ between the first and last nine trials of any condition (BO: $T_{+}=8.5, N=4, P=0.375$; $\mathrm{HV}: T_{+}=16.0, N=7, P=0.781$ ).

Focusing on trials in which subjects made a choice, we found that in the BO condition, orang-utans preferentially chose the tunnel at E's back (Wilcoxon signed-ranks test against 50\%; $T_{+}=73.0, N=12, P=0.005$; see Fig. 3 ). In the HV condition they chose the opaque tunnel marginally more (Wilcoxon signed-ranks test against $50 \% ; T_{+}=62.0, N=12, P=0.074$, and if one-tailed statistics are applied, as in Melis et al. 2006, this comparison reaches significance $(P=0.037))$. In the NS control, subjects showed no preference for either tunnel (Wilcoxon signed-ranks test against 50\%; $\left.T_{+}=38.5, N=11, P=0.659\right)$. We then directly compared subjects' preference for the tunnel at E's back in the body orientation condition and their preference for the opaque tunnel in the hidden-visible and nonsocial conditions. This comparison revealed no significant differences (Friedman test; $\chi^{2}=2.520, N=13$, $P=0.284$ ). A comparison of the orang-utans' preference in the first nine trials with their preference in the last nine trials did not reveal any significant changes for any of the conditions (Wilcoxon signedranks test; $\mathrm{BO}: T_{+}=32.0, N=10, P=0.676 ; \mathrm{HV}: T_{+}=52.0, N=11$, $P=0.098$; NS: $\left.T_{+}=37.5, N=10, P=0.336\right)$.

\section{Side Switches}

When $\mathrm{E}$ was present (i.e. in $\mathrm{BO}$ and $\mathrm{HV}$ ), the orang-utans sometimes switched sides before making their choice (see Table 2). This behaviour might have been caused by a general feeling of insecurity in the presence of $E$ or occurred because the orang-utans were deliberately changing their initial choice. In the former case the orang-utans should switch sides equally often in both 'social' conditions and irrespective of which tunnel they were initially inspecting. To rule out one or the other explanation, we therefore analysed the frequency of side switches across conditions as well as between both tunnels. Comparing the number of trials with at least one side switch, we found a significant difference between conditions (Friedman test: $\chi^{2}=15.622, \quad N=13$, $P<0.001)$. Post hoc comparisons revealed that side switches occurred most frequently in the HV condition compared with the other conditions (Wilcoxon signed-ranks test: HV-BO: $T_{+}=43.0$, $N=9, \quad P=0.016 ; \quad H V-N S: T_{+}=55.0, \quad N=10, \quad P=0.002$ ). Side switches were also more frequent in the BO than in the NS control $\left(T_{+}=28.0, N=7, P=0.016\right)$. Furthermore, the subjects switched sides depending on which tunnel they initially inspected. In the BO condition subjects were more likely to switch sides after initially 


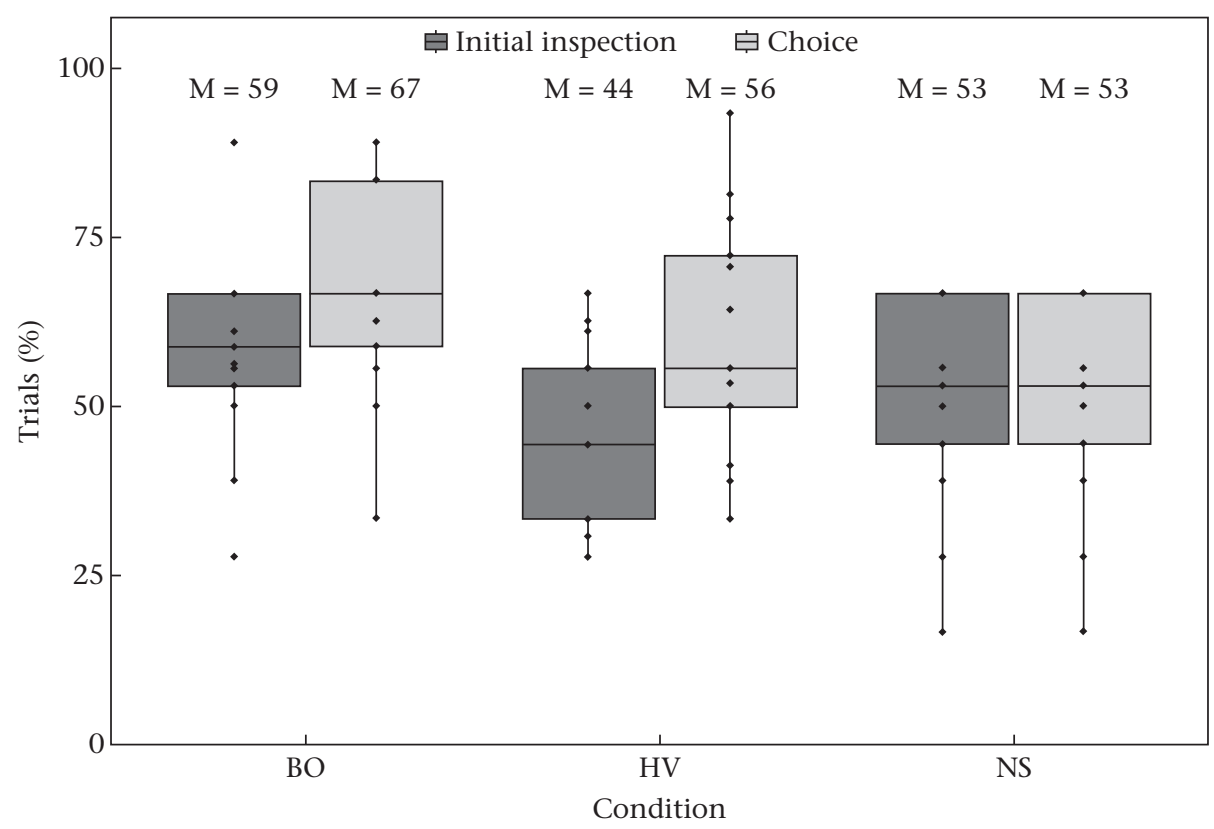

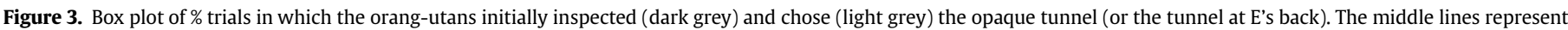

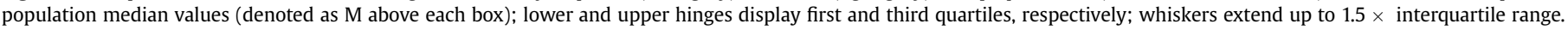
Filled black circles represent individual data points. BO: body orientation condition; HV: hidden-visible condition NS: nonsocial control.

inspecting the tunnel in front of $\mathrm{E}$ rather than after initially inspecting the tunnel at his back $\left(T_{+}=28.0, N=7, P=0.016\right)$. Likewise, subjects in the HV condition were more likely to switch sides after initially inspecting the transparent tunnel rather than after initially inspecting the opaque tunnel $\left(T_{+}=50.5, N=10\right.$, $P=0.016)$. The frequency of side switches did not differ between the first and last nine trials of any condition (BO: $T_{+}=10.5, N=6$, $\left.P=1 ; \mathrm{HV}: T_{+}=29.0, N=9, P=0.516\right)$.

\section{Initial Inspections}

If the orang-utans truly understood something about the competitor's perspective, the cues that were accessible from the starting position should have been sufficient for them to make a correct choice without first having to inspect any of the tunnels. Also, it might have been that some subjects initially inspected the correct tunnel but refrained from retrieving the reward because they were scared by the competitor's presence. Therefore, we additionally analysed whether the orang-utans had a tunnel preference when initially inspecting a particular side. In the BO condition, the orang-utans marginally preferred to initially inspect the tunnel at E's back significantly above chance (Wilcoxon signedranks test against $50 \% ; T_{+}=62.5, N=12, P=0.067$; see Fig. 3 ). Subjects did not show a preference in initially inspecting the opaque or the transparent tunnel, either in the HV condition (Wilcoxon signed-ranks test against $50 \% ; T_{+}=52.0, N=12, P=0.327$ ) or in the NS control (Wilcoxon signed-ranks test against $50 \% ; T_{+}=38.5$, $N=11, P=0.659$ ). Comparing a subject's preference to initially inspect the tunnel at E's back in the BO condition, with the preference to initially inspect the opaque tunnel in the HV and NS conditions, revealed no significant differences (Friedman test: $\chi^{2}=2.571, N=13, P=0.277$ ). Neither did we find any significant differences between the orang-utans' performance in the first nine trials and their performance in the last nine trials (Wilcoxon signed-ranks test; BO: $T_{+}=39.0, N=11, P=0.633$; HV: $T_{+}=18.0$, $N=6, P=0.156$; NS: $\left.T_{+}=37.5, N=10, P=0.336\right)$.

\section{Total Time of Initial Inspections}

Focusing on trials in which subjects made a choice without switching sides, we analysed how much time subjects spent with the initial inspection of the tunnel. There was a significant difference between conditions (Friedman test: $\chi^{2}=16.7692, N=13$, $P<0.001$; see Fig. 4). Post hoc comparisons revealed that initial inspection times were shortest in the NS control compared with the other conditions (Wilcoxon signed-ranks test: NS-BO: $T_{+}=90.0$, $N=13, P<0.001$; NS-HV: $\left.T_{+}=91.0, N=13, P<0.001\right)$. Mean initial inspection times did not differ significantly between the BO condition and the HV condition $\left(T_{+}=49.0, N=13, P=0.839\right)$. Next, we analysed whether initial inspection times differed depending on which tunnel was inspected. In the BO condition, subjects spent more time inspecting the tunnel in front of $E$ than inspecting the tunnel at E's back $\left(T_{+}=88.0, N=13, P=0.001\right)$. There was no significant difference between the time subjects spent inspecting the transparent and the opaque tunnel, either in the HV condition $\left(T_{+}=68.0, N=13, P=0.127\right)$, or in the NS control $\left(T_{+}=60.0\right.$, $N=13, P=0.340$ ). A comparison of the orang-utans' performance in the first and last nine trials revealed that subjects spent increasingly less time inspecting the tunnels in the NS control $\left(T_{+}=80.0, N=13, P=0.013\right)$, whereas inspection times did not change in the $\mathrm{BO}\left(T_{+}=50.0, N=13, P=0.685\right)$ and $\mathrm{HV}$ conditions $\left(T_{+}=50.0, N=13, P=0.787\right)$.

\section{DISCUSSION}

The first finding is that in the BO condition, the orang-utans clearly differentiated between the experimenter being oriented towards or away from the tunnels and they preferentially chose the tunnel at his back (even though they showed only a marginal preference in their initial inspections). Furthermore, they spent more time inspecting the tunnel in front of the experimenter than inspecting the tunnel at his back. This indicates that even when choosing 'wrongly' they were still sometimes sensitive to their impending failure. This is in line with other studies showing that 


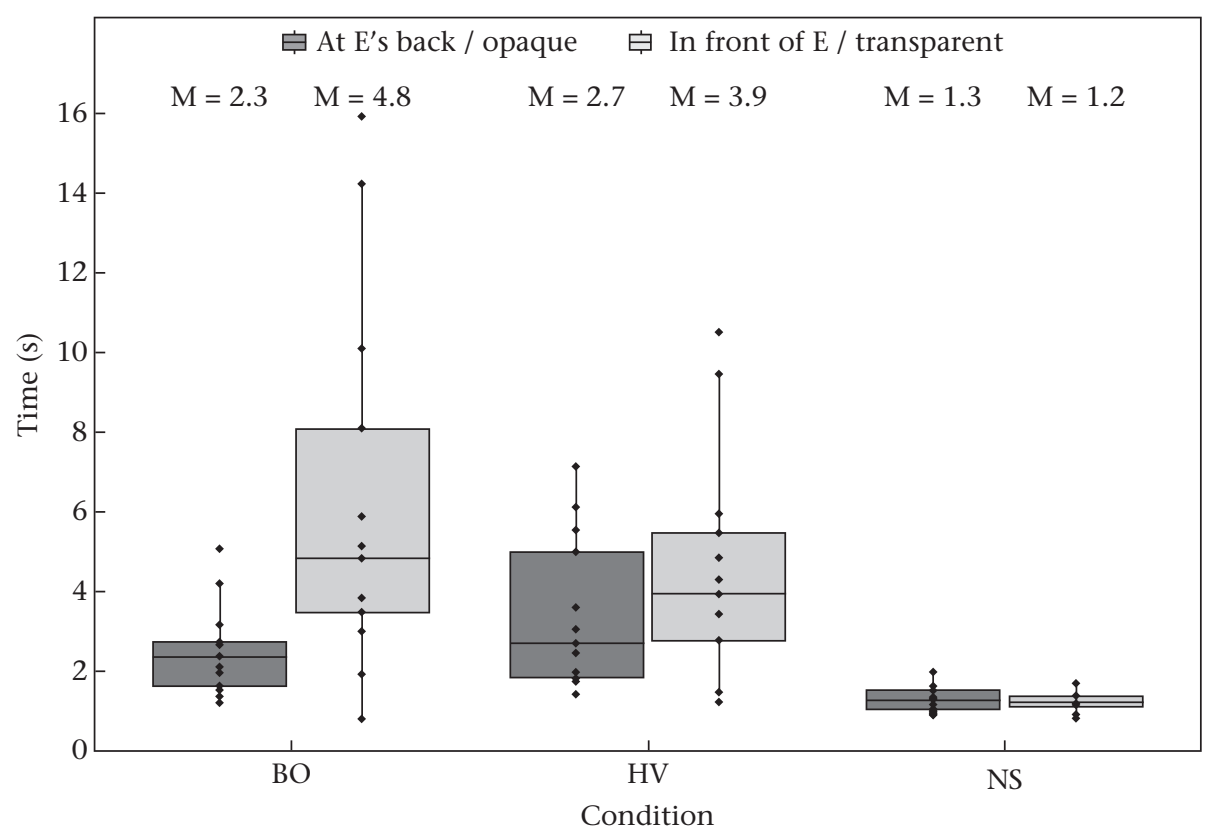

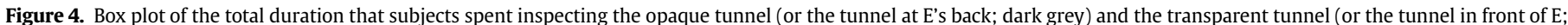

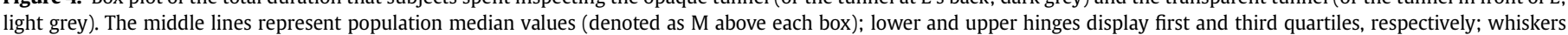

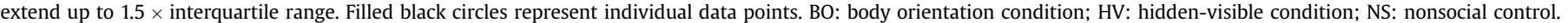

orang-utans can clearly differentiate others' attentional states (Kaminski et al. 2004; Liebal et al. 2004b, 2006; Poss et al. 2006; Tempelmann et al. 2011). This finding is, however, also the first to extend orang-utans' ability to determine others' attentional states to a competitive context.

The second finding is that in the crucial experimental condition during which the human was facing forwards, the orang-utans chose the opaque tunnel marginally more often than the transparent one. Orang-utans did not show any preference whatsoever if no competitor was present. This result is comparable to the result with the chimpanzees in Melis et al. (2006). However, contrary to the findings of Melis et al. a direct comparison of the performance between conditions revealed no significant differences, indicating that the effect is less clear-cut in orang-utans. Furthermore, in the current study the orang-utans employed a particular strategy before making their final choice. They frequently inspected the hole before making a choice and sometimes switched sides when they had initially inspected the transparent tunnel (notably, side switches from the opaque to the transparent tunnel were significantly less likely to occur). Furthermore, they sometimes refrained from opening the transparent tunnel (which was significantly likely to occur for the opaque tunnel). In their initial inspections the orang-utans did not show any preference for either tunnel. Furthermore, they were indifferent regarding the time they spent inspecting the opaque and the transparent tunnels before making their choice. This stands in contrast to their performance in the BO condition, where they hesitated longer before pulling open the flap in front of the experimenter, indicating that they lack any sensitivity to whether they had made a correct or an incorrect choice in the absence of BO cues.

These findings are difficult to reconcile with a full understanding of the human's perspective in the current situation. It could be that orang-utans' understanding of a human's perspective is limited and that they need salient cues (e.g. body posture) in order to determine whether or not a human can see them. If the orang-utans fully understand through which tunnel the human can or cannot see, we would expect them to make a choice from their starting point. Instead the orang-utans seem to make their choice after arriving at one of the tunnels, and sometimes decide to switch sides before reaching for the food.

One explanation for this result could be that even though all attempts were made to ensure that the subjects could not see the human from either tunnel, the orang-utans were still able to glimpse the human from the transparent (but not the opaque) tunnel. Therefore it could be that rather than understanding the human's perspective, the orang-utans used an egocentric strategy of inspecting whether the human could be seen from their current side (e.g. the transparent tunnel). If they managed to see the human through the tunnel, they either decided to switch to the other side or refrained from opening the tunnel. We found no increase in performance in any of the behavioural measures in the HV condition, suggesting that this egocentric rule was not adopted throughout the study (e.g. by associative learning). Also, as the orang-utans did not show any preference for the opaque tunnel if no competitor was present, it can be ruled out that their behaviour was driven by a general tendency to retrieve food from visually occluded places (especially as both rewards were actually placed in the open space). Still, the pure presence of the competitor might have been sufficient to trigger this kind of behavioural rule, causing them to follow it without further consideration.

An alternative explanation for the orang-utans' differing performance in the $\mathrm{BO}$ and the $\mathrm{HV}$ condition might be that the $\mathrm{BO}$ condition was virtually identical to the warm-up trials (except for some added visual barriers). Therefore, the possibility remains that the orang-utans simply learned during the warm-up to associate the reward with the experimenter's back as an arbitrary connection with no understanding of another individual's attention at all. However, if this were the case, it is unlikely that the subjects learned this solely during the warm-up phase. As some studies have demonstrated, apes perform poorly in exploiting arbitrary cues to locate hidden food (e.g. in associating a white bottle with a food reward; see Hanus \& Call 2011). As the number of trials presented during warm-up would have been insufficient to newly acquire a previously arbitrary cue, we rather think that the cue of 
body orientation was already a meaningful stimulus for the orangutans before they participated in the study at hand. Moreover, as our subjects were used to being fed by their human keepers, they experienced a relatively low level of direct food competition (and if anything were more likely to associate the front of humans with food reward).

In summary, our results suggest that orang-utans may have only a limited understanding of others' perspectives, relying mainly on salient cues such as facial and bodily orientation and body posture. Melis et al. (2006) did not report whether and how often their subjects switched sides before making a choice, and therefore it cannot be completely ruled out that they employed the same strategy as the orang-utans. However, in contrast to chimpanzees, for whom a plethora of evidence suggests a sophisticated understanding of others' perspectives, the current results are in line with evidence from other studies that have indicated less distinctive gaze following (Okamoto-Barth et al. 2007) and lower sensitivity to visual attention cues (Liebal et al. 2004b) in orang-utans than in the genus Pan. Okamoto-Barth et al. (2007) found orang-utans, in contrast to the other great ape species, to be insensitive to whether a barrier was opaque or had a window when tracking the gaze of a human experimenter to a target location. In the study of Liebal et al. (2004b), orang-utans requesting food from a human experimenter who had turned around, leaving the food behind them, also frequently gestured out of sight behind the experimenter's back. These, and the current results, seem to be at odds with the findings of Shillito et al. (2005), who reported a single female orang-utan to be highly sensitive to the attentional state of a human while directing the human to desirable objects out of the subject's reach. In this task the orang-utan had to discriminate between two experimenters, one wearing a bucket over his head and the other with an unimpeded view. Like the orang-utans in the HV condition of the present study, the orang-utan could not solve the task by relying on cues from body posture, as the humans were always oriented towards her. However, in contrast to the orang-utans in our study, she could solve the task egocentrically by basing her judgement on the humans' eyes, which are known to be a very salient cue for attention (reviewed in Emery 2000). Note that Kaminski et al. (2004) also found two orang-utans that adjusted their food requests according to whether a human had her eyes open or shut. In addition, the orang-utan in Shillito et al.'s study failed to discriminate between opaque and transparent visual barriers, a distinction that our subjects essentially needed to make in order to successfully retrieve the food in the HV condition.

Although the sophisticated perspective-taking skills of corvids and chimpanzees presumably represent a case of convergent evolution (Emery \& Clayton 2004), the limited level of perspective taking in orang-utans, compared with that of chimpanzees (and humans), suggests that within the great ape clade this skill may have increased gradually after the human lineage split from our common ancestor. However, it might also be the case that orangutans gradually lost this trait throughout the course of evolution as a result of their more solitary lifestyle. For instance, ringtailed lemurs, Lemur catta, have been found to perform better in gaze following and attention reading tasks than closely related lemur species living in less complex social groups (Sandel et al. 2011). Notably, rhesus macaques, Macaca mulatta, competing with a human for food in a series of studies very similar to the present one (Flombaum \& Santos 2005; Santos et al. 2006), also took into account what others could see (and hear). However, similarly to Shilitio et al.'s study, the macaques might have used the visibility of the human's eyes and gaze direction as discriminative cues to solve the task. Thus, it is possible that the rhesus monkeys were simply avoiding food that had been looked at by a human (see Burkart \& Heschl 2007 for a similar interpretation for marmosets, Callithrix jacchus, competing against dominant conspecifics for food). Furthermore, there is only little evidence so far that social complexity was a driving factor in the evolution of avian cognition (Emery 2004; but see also Bond et al. 2003, 2007). Therefore, another possibility might be that high levels of direct food competition might have driven the development of more sophisticated perspective-taking skills in chimpanzees and corvids, whereas for a species such as orang-utans with less frequent direct competition for food resources a limited understanding of others perspectives might be sufficient (note, however, that differences from the chimpanzees' performance in Melis et al. (2006) cannot be explained by motivational reasons, as the orang-utans in the present study were nevertheless generally willing and motivated to compete, as demonstrated by their performance in the BO condition). As yet, the evidence available from different species is too patchy and incomplete to decide on one of these alternative evolutionary trajectories. Future studies, investigating the perspective-taking skills of a wider variety of different species with various experimental paradigms will be required to fill this gap. Regarding the great ape clade, studies on gorillas (due to their reduced genetic distance from the Pan genera and humans) and Sumatran orang-utans, Pongo abelii, who have been reported to be more sociable than their Bornean counterparts (Delgado \& van Schaik 2000), might be especially illuminating.

\section{Acknowledgments}

Many thanks to Biruté M. F. Galdikas and the staff at the Orangutan Care Centre and Quarantine in Pasir Panjang for their support as well as to the Indonesian Ministry of Research and Technology (RISTEK) for granting us permission to do research in their country. We thank Antje Girndt for reliability coding, Johannes Großmann for help in constructing the tunnel apparatuses, Roger Mundry for statistical advice and Nathan Pyne-Carter for the perusal of a former version of the manuscript and helpful comments. This research is part of the project 'Evolutionary Roots of Human Social Interaction (ROSI)' funded by the VolkswagenStiftung, Germany. The reported experiments comply with all laws of the country in which they were performed.

\section{References}

Amici, F., Aureli, F., Visalberghi, E. \& Call, J. 2009. Spider monkeys (Ateles geoffroyi) and capuchin monkeys (Cebus apella) follow gaze around barriers: evidence for perspective taking? Journal of Comparative Psychology, 123, 368-374.

von Bayern, A. M. P. \& Emery, N. J. 2009. Jackdaws respond to human attentional states and communicative cues in different contexts. Current Biology, 19, 602-606

Bolker, B. M., Brooks, M. E., Clark, C. J., Geange, S. W., Poulsen, J. R., Stevens, M. H. H. \& White, J-S. S. 2009. Generalized linear mixed models: a practical guide for ecology and evolution. Trends in Ecology and Evolution, 24, 127-135.

Bond, A. B., Kamil, A. C. \& Balda, R. P. 2003. Social complexity and transitive inference in corvids. Animal Behaviour, 65, 479-487.

Bond, A. B., Kamil, A. C. \& Balda, R. P. 2007. Serial reversal learning and the evolution of behavioral flexibility in three species of north American corvids (Gymnorhinus cyanocephalus, Nucifraga columbiana, Aphelocoma californica). Journal of Comparative Psychology, 121, 372-379.

Bräuer, J., Call, J. \& Tomasello, M. 2004. Visual perspective taking in dogs (Canis familiaris) in the presence of barriers. Applied Animal Behaviour Science, 88, 299-317.

Bräuer, J., Call, J. \& Tomasello, M. 2005. All great ape species follow gaze to distant locations and around barriers. Journal of Comparative Psychology, 119, 145-154.

Bräuer, J., Call, J. \& Tomasello, M. 2007. Chimpanzees really know what others can see in a competitive situation. Animal Cognition, 10, 439-448.

Bugnyar, T. 2011. Knower-guesser differentiation in ravens: others' viewpoints matter. Proceedings of the Royal Society B, 278, 634-640.

Bugnyar, T. \& Heinrich, B. 2005. Ravens, Corvus corax, differentiate between knowledgeable and ignorant competitors. Proceedings of the Royal Society B, 272, 1641-1646. 
Bugnyar, T., Stöwe, M. \& Heinrich, B. 2004. Ravens, Corvus corax, follow gaze direction of humans around obstacles. Proceedings of the Royal Society B, 271, 1331-1336.

Burkart, J. M. \& Heschl, A. 2007. Understanding visual access in common marmosets, Callithrix jacchus: perspective taking or behaviour reading? Animal Behaviour, 73, 457-469.

Call, J., Hare, B. \& Tomasello, M. 2001. Gaze following in five non-human primate species. Folia Primatologica, 72, 347-348.

Call, J., Bräuer, J., Kaminski, J. \& Tomasello, M. 2003. Domestic dogs (Canis familiaris) are sensitive to the attentional state of humans. Journal of Comparative Psychology, 117, 257-263.

Carter, J., Lyons, N. J., Cole, H. L. \& Goldsmith, A. R. 2008. Subtle cues of predation risk: starlings respond to a predator's direction of eye-gaze. Proceedings of the Royal Society B, 275, 1709-1715.

Dally, J. M., Emery, N. J. \& Clayton, N. S. 2004. Cache protection strategies by western scrub-jays (Aphelocoma californica): hiding food in the shade. Proceedings of the Royal Society B, 271, S387-S390.

Dally, J. M., Emery, N. J. \& Clayton, N. S. 2006. Food-caching western scrub-jays keep track of who was watching when. Science, 310, 1662-1665.

Delgado, R. A., Jr. \& van Schaik, C. P. 2000. The behavioral ecology and conservation of the orangutan (Pongo pygmaeus): a tale of two islands. Evolutionary Anthropology, 9, 201-218.

Emery, N. J. 2000. The eyes have it: the neuroethology, function and evolution of social gaze. Neuroscience E' Biobehavioral Reviews, 24, 581-604.

Emery, N. J. 2004. Are corvids 'feathered apes'? Cognitive evolution in crows, jays, rooks and jackdaws. In: Comparative Analysis of Minds (Ed. by S. Watanabe) pp. 181-213. Tokyo: Keio University Press.

Emery, N. J. \& Clayton, N. S. 2001. Effects of experience and social context on prospective caching strategies by scrub jays. Nature, 414, 443-446.

Emery, N. J. \& Clayton, N. S. 2004. The mentality of crows: convergent evolution of intelligence in corvids and apes. Science, 306, 1903-1907.

Flavell, J. H., Everett, B. A., Croft, K. \& Flavell, E. R. 1981. Young children's knowledge about visual perception: further evidence for the level 1 level 2 distinction. Developmental Psychology, 17, 99-103.

Flombaum, J. I. \& Santos, L. R. 2005. Rhesus monkeys attribute perceptions to others. Current Biology, 15, 447-452.

Gácsi, M., Miklósi, Á., Varga, O., Topál, J. \& Csányi, V. 2004. Are readers of our face readers of our minds? Dogs (Canis familiaris) show situation-dependent recognition of human's attention. Animal Cognition, 7, 144-153.

Hanus, D. \& Call, J. 2011. Chimpanzee problem-solving: contrasting the use of causal and arbitrary cues. Animal Cognition, 14, 871-878.

Hare, B. 2001. Can competitive paradigms increase the validity of experiments on primate social cognition? Animal Cognition, 4, 269-280.

Hare, B., Call, J. \& Tomasello, M. 2001. Do chimpanzees know what conspecifics know? Animal Behaviour, 61, 139-151.

Hare, B., Call, J. \& Tomasello, M. 2006. Chimpanzees deceive a human competitor by hiding. Cognition, 101, 495-514.

Hattori, Y., Kuroshima, H. \& Fujita, K. 2010. Tufted capuchin monkeys (Cebus apella) show understanding of human attentional states when requesting food held by a human. Animal Cognition, 13, 87-92.

Hostetter, A. B., Cantero, M. \& Hopkins, W.D. 2001. Differential use of vocal and gestura communication by chimpanzees (Pan troglodytes) in response to the attentional status of a human (Homo sapiens). Journal of Comparative Psychology, 115, 337-343.

Hostetter, A. B., Russell, J. L., Freeman, H. \& Hopkins, W. D. 2007. Now you see me, now you don't: evidence that chimpanzees understand the role of the eyes in attention. Animal Cognition, 10, 55-62.

Kaminski, J., Call, J. \& Tomasello, M. 2004. Body orientation and face orientation: two factors controlling apes' begging behavior from humans. Animal Cognition, 7, 216-223.

Kaminski, J., Call, J. \& Tomasello, M. 2006. Goats' behaviour in a competitive food paradigm: evidence for perspective taking? Behaviour, 143, 1341-1356.

Kaminski, J., Call, J. \& Tomasello, M. 2008. Chimpanzees know what others know, but not what they believe. Cognition, 109, 224-234.

Kaminski, J., Riedel, J., Call, J. \& Tomasello, M. 2005. Domestic goats, Capra hircus, follow gaze direction and use social cues in an object choice task. Animal Behaviour, 69, 11-18.
Krützen, M., Willems, Erik P. \& van Schaik, Carel P. 2011. Culture and geographic variation in orangutan behavior. Current Biology, 21, 1808-1812.

Leavens, D., Russell, J. \& Hopkins, W. 2010. Multimodal communication by captive chimpanzees (Pan troglodytes). Animal Cognition, 13, 33-40.

Liebal, K., Pika, S. \& Tomasello, M. 2004a. Social communication in siamangs (Symphalangus syndactylus): use of gestures and facial expressions. Primates, 45, 41-57.

Liebal, K., Pika, S. \& Tomasello, M. 2006. Gestural communication of orangutans (Pongo pygmaeus). Gesture, 6, 1-38.

Liebal, K., Pika, S., Call, J. \& Tomasello, M. 2004b. To move or not to move: how great apes adjust to the attentional state of others. Interaction Studies, 5, 199-219.

Locke, D. P., Hillier, L. W., Warren, W. C., Worley, K. C., Nazareth, L. V., Muzny, D. M., Yang, S. P., Wang, Z., Chinwalla, A. T., Minx, P., et al. 2011. Comparative and demographic analysis of orang-utan genomes. Nature, $\mathbf{4 6 9}$, 529-533.

Loretto, M.-C., Schloegl, C. \& Bugnyar, T. 2010. Northern bald ibises follow others' gaze into distant space but not behind barriers. Biology Letters, 6, 14-17.

Melis, A. P., Call, J. \& Tomasello, M. 2006. Chimpanzees (Pan troglodytes) conceal visual and auditory information from others. Journal of Comparative Psychology, 120, 154-162.

Moll, H. \& Meltzoff, A. N. 2011. How does it look? Level 2 perspective-taking at 36 months of age. Child Development, 82, 661-673.

Okamoto-Barth, S., Call, J. \& Tomasello, M. 2007. Great apes' understanding of other individuals' line of sight. Psychological Science, 18, 462-468.

Pika, S. \& Bugnyar, T. 2011. The use of referential gestures in ravens (Corvus corax) in the wild. Nature Communications, 2, 560.

Poss, S. R., Kuhar, C., Stoinski, T. S. \& Hopkins, W. D. 2006. Differential use of attentional and visual communicative signaling by orangutans (Pongo pygmaeus) and gorillas (Gorilla gorilla) in response to the attentional status of a human. American Journal of Primatology, 68, 978-992.

Povinelli, D. J. \& Eddy, T. J. 1996. Factors influencing young chimpanzees' (Pan troglodytes) recognition of attention. Journal of Comparative Psychology, 110, 336-345.

Proops, L. \& McComb, K. 2010. Attributing attention: the use of human-given cues by domestic horses (Equus caballus). Animal Cognition, 13, 197-205.

Range, F. \& Virányi, Z. 2011. Development of gaze following abilities in wolves (Canis lupus). PLoS ONE, 6, e16888.

Rosati, A. G. \& Hare, B. 2009. Looking past the model species: diversity in gazefollowing skills across primates. Current Opinion in Neurobiology, 19, 45-51.

Sandel, A. A., MacLean, E. L. \& Hare, B. 2011. Evidence from four lemur species that ringtailed lemur social cognition converges with that of haplorhine primates. Animal Behaviour, 81, 925-931.

Santos, L. R., Nissen, A. G. \& Ferrugia, J. A. 2006. Rhesus monkeys, Macaca mulatta, know what others can and cannot hear. Animal Behaviour, 71, 1175-1181.

van Schaik, C. P. 1999. The socioecology of fission-fusion sociality in orangutans. Primates, 40, 69-86.

van Schaik, C. P., Ancrenaz, M., Borgen, G., Galdikas, B., Knott, C. D., Singleton, I., Suzuki, A., Utami, S. S. \& Merrill, M. 2003. Orangutan cultures and the evolution of material culture. Science, 299, 102-105.

Shillito, D. J., Shumaker, R. W., Gallup, G. G., Jr. \& Beck, B. B. 2005. Understanding visual barriers: evidence for level 1 perspective taking in an orang-utan, Pongo pygmaeus. Animal Behaviour, 69, 679-687.

Sugardjito, J., te Boekhorst, I. \& van Hooff, J. 1987. Ecological constraints on the grouping of wild orang-utans (Pongo pygmaeus) in the Gunung Leuser National Park, Sumatra, Indonesia. International Journal of Primatology, 8, 17-41.

te Boekhorst, I. J. A., Schürmann, C. L. \& Sugardjito, J. 1990. Residential status and seasonal movements of wild orang-utans in the Gunung Leuser Reserve (Sumatra, Indonesia). Animal Behaviour, 39, 1098-1109.

Tempelmann, S., Kaminski, J. \& Liebal, K. 2011. Focus on the essential: all great apes know when others are being attentive. Animal Cognition, 14, 433-439.

Wilkinson, A., Mandl, I., Bugnyar, T. \& Huber, L. 2010. Gaze following in the redfooted tortoise (Geochelone carbonaria). Animal Cognition, 13, 765-769.

Xitco, M. J., Gory, J. D. \& Kuczaj, S. A. 2004. Dolphin pointing is linked to the attentional behavior of a receiver. Animal Cognition, 7, 231-238.

Zimmermann, F., Zemke, F., Call, J. \& Gómez, J. C. 2009. Orangutans (Pongo pygmaeus) and bonobos (Pan paniscus) point to inform a human about the location of a tool. Animal Cognition, 12, 347-358. 